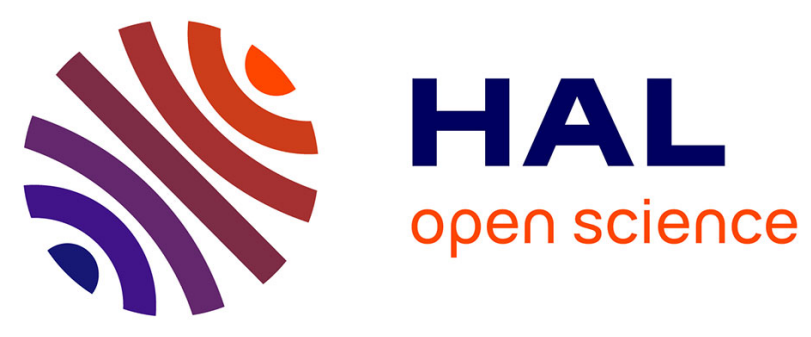

\title{
Media Ecosystems: A Novel Approach for Content-Awareness in Future Networks
}

Harilaos Koumaras, Daniel Negru, Eugen Borcoci, Vaios Koumaras, Kostas

Troulos, Yael Lapid, Evangelos Pallis, Mamadou Sidibé, Antonio Pinto, George Gardikis, et al.

\section{To cite this version:}

Harilaos Koumaras, Daniel Negru, Eugen Borcoci, Vaios Koumaras, Kostas Troulos, et al.. Media Ecosystems: A Novel Approach for Content-Awareness in Future Networks. Springer. The Future Internet Lecture Notes in Computer Science Volume 6656, 2011, pp 369-380, Springer, pp.369-380, 2011, Lecture Notes in Computer Science, 978-3-642-20898-0. 10.1007/978-3-642-20898-0_26 . hal00998555

\section{HAL Id: hal-00998555 https://hal.science/hal-00998555}

Submitted on 2 Jun 2014

HAL is a multi-disciplinary open access archive for the deposit and dissemination of scientific research documents, whether they are published or not. The documents may come from teaching and research institutions in France or abroad, or from public or private research centers.
L'archive ouverte pluridisciplinaire HAL, est destinée au dépôt et à la diffusion de documents scientifiques de niveau recherche, publiés ou non, émanant des établissements d'enseignement et de recherche français ou étrangers, des laboratoires publics ou privés. 


\title{
Media Ecosystems: A Novel Approach for Content- Awareness in Future Networks
}

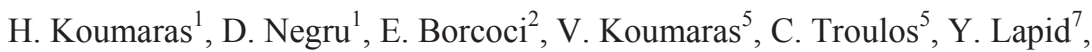 \\ E. Pallis ${ }^{8}$, M. Sidibé ${ }^{6}$, A. Pinto ${ }^{9}$, G. Gardikis ${ }^{3}$, G. Xilouris ${ }^{3}$, and C. Timmerer ${ }^{4}$ \\ ${ }^{1}$ CNRS LaBRI laboratory, University of Bordeaux, France \\ koumaras@ieee.org, daniel.negrullabri.fr \\ ${ }^{2}$ Telecommunication Dept., University Politehnica of Bucharest (UPB), Romania \\ eugen.borcoci@elcom.pub.ro \\ ${ }^{3}$ Institute of Informatics and Telecommunications, NCSR Demokritos, Greece \\ \{gardikis, xilouris\}@iit.demokritos.gr \\ ${ }^{4}$ Multimedia Communication, Klagenfurt University, Austria \\ christian.timmerer@itec.uni-klu.ac.at \\ ${ }^{5} \mathrm{PCN}$, Greece \\ vkoumaras@pcngreece.com, ktroulos@pcngreece.com \\ ${ }^{6}$ VIOTECH Communications, France \\ msidibe@viotech. net \\ ${ }^{7}$ Optibase Technologies Ltd, Israel \\ Yaell@optibase.com \\ ${ }^{8}$ Applied Informatics and Multimedia Dept., TEI of Crete, Greece \\ pallis@pasiphae.teiher.gr \\ ${ }^{9}$ INESC Porto, Portugal \\ apinto@inescporto.pt
}

\begin{abstract}
This chapter proposes a novel concept towards the deployment of a networked 'Media Ecosystem'. The proposed solution is based on a flexible cooperation between providers, operators, and end-users, finally enabling every user first to access the offered multimedia services in various contexts, and second to share and deliver his own audiovisual content dynamically, seamlessly, and transparently to other users. Towards this goal, the proposed concept provides content-awareness to the network environment, network- and user contextawareness to the service environment, and adapted services/content to the end user for his best service experience possible, taking the role of a consumer and/or producer.
\end{abstract}

Keywords: Future Internet, Multimedia Distribution, Content Awareness, Network Awareness, Content/Service Adaptation, Quality of Experience, Quality of Services, Service Composition, Content-Aware Network

\section{$1 \quad$ Introduction}

One of the objectives of the future communication networks is the provision of audiovisual content in flexible ways and for different contexts, at various quality standards and associated price levels. The end user (EU) is ultimately interested in getting a 
good Quality of Experience (QoE) at convenient prices. QoE is defined in [15] as "the overall acceptability of an application or service, as perceived subjectively by the end user". Therefore, QoE concepts are considered as important factors in designing multimedia distribution systems. The system capabilities to assure different levels of endto-end Quality of Services, (i.e. including content production, transport and distribution) together with the possibility to evaluate and react to the QoE level at user terminals, offer to the EU a wide range of potential choices, covering the possibilities of low, medium or high quality levels.

Towards that direction, three factors (technologies) can be considered and can cooperate in an integrated system:

- First, significant advances in digital video encoding and compression techniques are now available. These techniques can achieve high compression ratios by exploiting both spatial and temporal redundancy in video sequences; and provides means for storage, transmission, and provision of very high-volume video data.

- Second, the development of advanced networking technologies in the access and core parts, with QoS assurance is seen. A flexible way of usage - based on virtualised overlays - can offer a strong support for the transportation of multimedia flows.

- Third, the todays' software technologies support the creation and composition of services while being able to take into account information regarding the transport/terminal contexts and adapt the services accordingly.

Bringing together in a synergic way all the above factors, a new "Media Ecosystem" is hence foreseen to arise, gathering a mass of not only existing but also new potential content distributors and media service providers, promoting passive consumers to engaged content creators. Such a "Media Ecosystem" is proposed in ALICANTE FP7 project [1-2]. By analogy to the ecology or market settings, a Media Ecosystem can be defined by inter-working environments, to which various actors belong to and through which they collaborate, in the networked media domain. With this respect, new flexible business models have to be supported by the Media Ecosystem. Finally, such a system can bring breakthrough opportunities in various domains such as communication industry, education, culture and entertainment.

However, the traditional and current layered architectures do not include exchanges of content - and network-based information between the network layers and upper layers. So, the added value of such interactions has not yet been exploited to better support the QoS and QoE requirements that media consumers are placing today. Network neutrality has been the foundational principle of the Internet, albeit today is revisited by service providers, research communities and industry, as a mean for quality provision and profit, to allow sustainable new forms of multimedia communications with an increasing importance in the Future Internet.

This suggests that the emerging approach of Content-Aware Networks (CAN) and Network-Aware services/Applications (NAA) can be a way to overcome the traditional architectures limitations. The network provisions based on CAN concepts, cooperating with powerful media adaptation techniques embedded in the network nodes, can be a foundation for an user-centric approach (i.e. satisfying the different 
needs of individual users) or service-centric approach (i.e. satisfying the different needs of various service types), that is required for the future services and applications [1-2]. The new CAN/NAA concept no more supposes network neutrality, but more intelligence and a higher degree of coupling to the upper layers are embedded in the network nodes. Based on virtualization, the network can offer enhanced transport and adaptation-capable services.

This chapter will introduce and describe an advanced architecture and new functionalities for efficient cooperation between entities of various environments so as to finally provide the end user with the best and most complete service experience via a Media Ecosystem, aiming to provide content-awareness to the network environment, network- and user context-awareness to the service environment, and adapted services/content to the end user's Environment.

\section{Background}

Numerous events and studies are currently dedicated to (re)define the directions which the Future Internet development should follow. Among other issues, a higher coupling between application and network layers are investigated, targeting to better performance (for multimedia) but without losing modularity of the architecture. [311]. The CAN/NAA approach can naturally lead to a user-centric infrastructure and telecommunication services as described in [3]. The strong orientation of user-centric awareness to services and content is emphasized in [4]. The works [5-6] consider that CAN/NAA can offer a way for evolution of networks beyond IP, while QoE issues related to *-awareness are discussed in [7]. The capability of content-adaptive network awareness is exploited in [8] for joint optimization of video transmission. The architecture can be still richer if to content awareness we add context awareness, [9]. The virtualisation as a powerful tool to overcome the Internet ossification by creating overlays is discussed in [10-11]. Finally, [14] discusses research challenges and open issues when adopting Scalable Video Coding (SVC) as a tool for CANs.

\section{System Architecture}

The proposed Media Ecosystem in ALICANTE project [1-2], by analogy with the ecology or business counterparts, can be characterized by inter-working environments to which the actors belong and through which they collaborate, in the networked media domain. These environments are:

- User Environment (UE), to which the end users belong;

- Service Environment (SE), to which the service and content providers belong;

- Network Environment (NE), to which the network providers belong.

By Environment, it is understood a generic and comprehensive name to emphasize a grouping of functions defined around the same functional goal and possibly spanning, vertically, one or more several architectural (sub-)layers. It characterizes a broader scope with respect to the term layer. By Service, if not specified differently, we understand here high level services, as seen at application/service layer. 


\subsection{Layered Architectural Model}

The ALICANTE architecture contains vertically several environments/layers and can be horizontally spanned over multiple network domains.

The User Environment (UE) includes all functions related to the discovery, subscription, consumption of the services by the EUs. At the Service Environment (SE) the Service Provider (SP) entity is the main coordinator. The architecture can support both synchronous communications or publish/subscribed ones. A novel type of service registry with enhanced functionalities allows new services supporting a variety of use case scenarios. Rich service composition in various ways is offered to EUs, opening them the role of SP/CP and manager. User and service mobility is also targeted.

Below the SE there is a new Home-Box (HB) layer to coordinate the actual content delivery to the end user's premises. The HB layer aims at allowing SPs to supply users with advanced context-aware multimedia services in a consistent and interoperable way. It enables uniform access for heterogeneous terminals and supports enhanced Quality of Experience (QoE). At the HB layer, the advanced user context management and monitoring functions provides real-time information on user context and network conditions, allowing better control over multimedia delivery and intelligent adaptation. The assembly of HBs is called layer because the HBs can logically communicate with each other in both client/server style but also in peer-to-peer (P2P) style. The HBs are located at the edges of the Content Consumer (CC) premises and have important roles in distribution of media flows, adaptation, routing, media caching, security, etc. Thus, the HB, which can be seen as the evolution of today's Home Gateway, is a physical entity aimed to be deployed inside each user's home.

The Network Environment (NE) comprises the virtual CAN layer (on top) and the traditional network infrastructure layer (at the bottom). The virtual CANs can span a single or multiple core network domains having content aware processing capabilities in terms of QoS, monitoring, media flow adaptation, routing/forwarding and security. The goal of the Virtual CAN layer is to offer to higher layers enhanced connectivity services, based on advanced capabilities (1) for network level provisioning of resources in a content-aware fashion and (2) for applying reactive adaptation measures based on media intelligent per flow adaptation. Innovative components, instantiating the CAN are called Media-Aware Network Elements (MANE). They are actually CANenabled routers, which together with the associated managers and the other elements of the ecosystem, offer content- and context-aware Quality of Service/Experience, adaptation, security, and monitoring features. The set of MANEs form together a Virtual Content-Aware Network, which will be denoted VCAN or simply CAN where no ambiguity exist.

ALICANTE's advanced concept provides adapted services/content to the end-user for her/his best service experience possible. The adaptation is provided at both the $\mathrm{HB}$ and CAN layers, and is managed by the Adaptation Decision-Taking Framework $($ ADTF $)$ - deployed at both layers. The ADTF is metadata-driven, thus enabling dynamic adaptation based on context information. The adaptation decisions will be taken based on three families of parameters: user preferences, terminal capabilities 
and network conditions. These parameters are gathered from every environment using dedicated user profile management and/or monitoring entities/subsystems.

The adaptation deployed at the CAN layer will be performed in the Media-Aware Network Elements (MANE): MANEs, which receive feedback messages about the terminal capabilities and channel conditions, can remove the non-required parts from a scalable bitstream before forwarding it. Thus, the loss of important transmission units due to congestion can be avoided and the overall error resilience of the video transmission service can be substantially improved. That is, within the CAN layer only scalable media formats - such as SVC - are delivered adopting a layeredmulticast approach which allows the adaptation of scalable media resources by the MANEs implementing the concept of distributed adaptation. In this way, all actors within the media ecosystem will benefit from this approach ranging from providers (i.e., service, content, network) to consumers/end users.

At the border to the user, i.e., the Home-Box, adaptation modules are deployed enabling device-independent access to the SVC-encoded content by providing X-toSVC and SVC-to-X transcoding functions with $\mathrm{X}=\{$ MPEG-2, MPEG-4 Visual, MPEG-4 AVC, etc.\}. An advantage of this approach is the reduction of the load on the network (i.e., no duplicates), making it free for (other) data (e.g., more enhancement layers).

The key innovations of this approach to service/content adaptation are - distributed, self organizing adaptation decision-taking framework; distributed dynamic and intelligent adaptation at HB and CAN layers; efficient, scalable SVC tunnelling and signalling thereof, and as result - high impact on the Quality of Service/Experience (QoS/QoE).

Figure 1 presents a high level view of the general Media Ecosystem layered architecture.

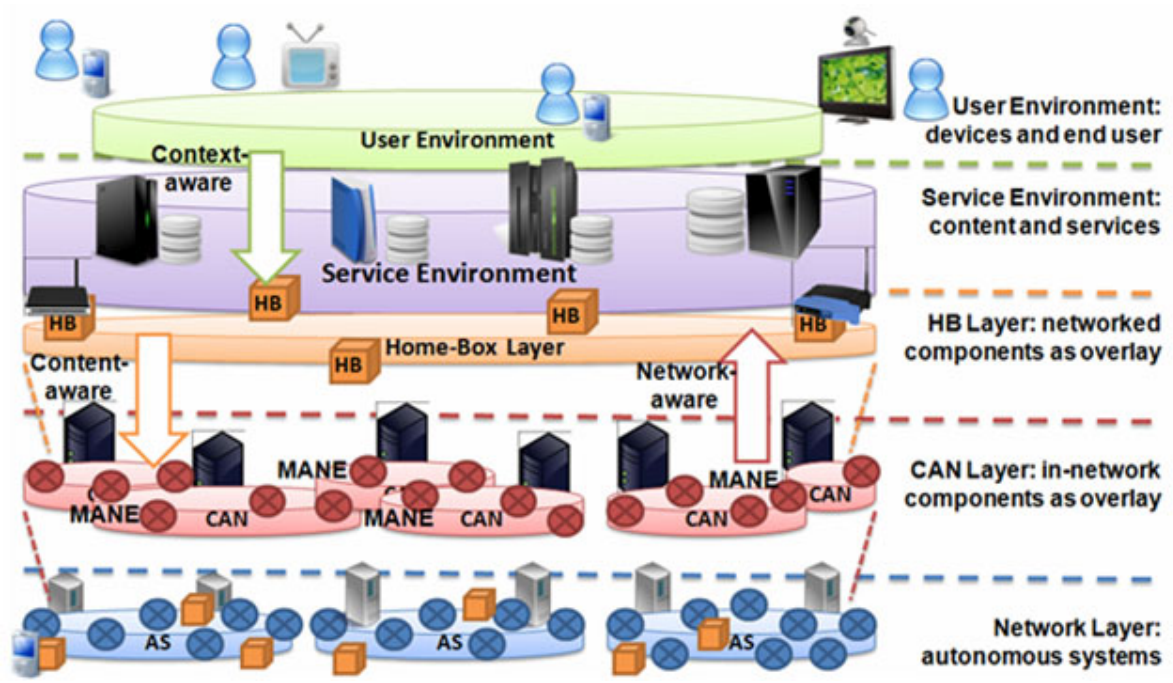

Fig. 1. ALICANTE general architecture - high level view 
To preserve each NP independency - which is an important real world need - the ALICANTE solution considers, for each network domain the existence of an Intradomain Network Resource Manager (NRM). This is responsible for the actual routers configuration in its own network, based on cooperation with the CAN Manager (CANMgr ) belonging to the CAN Provider (CANP). Each domain can accommodate several CANs.

The system architecture can be horizontally divided into: Management, Control and Data Planes (MPl, CPl, DPl), parallel and cooperating (not represented explicitly in the picture). The upper data plane interfaces at the CAN layer and transport the packets between the VCAN layer and the Home-Box layer in both directions. The downloaded packets are especially marked by the application layer, allowing association with the correct CAN.

\subsection{Content-Aware Networks (CAN)}

The SPs may request the CANP to create multi-domain VCANs in order to benefit from different purposes (content-aware forwarding, QoS, security, unicast/multicast, etc.). The architecture supports creation of parallel VCANs over the same network infrastructure. Specialization of VCANs may exist (content-type aware), in terms of forwarding, QoS level of guarantees QoS granularity, content adaptation procedures, degree of security, etc. The amount of VCAN resources can be changed during network functioning based on monitoring developed at CAN layer and conforming to a contract (SLA) previously concluded between the SP and CANP. From deployment point of view the ALICANTE solution is flexible, specifically an evolutionary approach is possible, i.e. to still keep a single control-management plane, while a more radical approach can also be envisaged towards full virtualization (i.e. independent management and control per VCAN).

The media data flows, are intelligently classified at ingress MANEs, and associated to the appropriate VCANs in order to be processed accordingly based on: (1) protocol header and metadata analysis (in-band information), (2) based on information delivered by the VCAN management, and (3) statistical information in the packet flows. Then, it will assign the flows to the appropriate CANs. Solutions to maximise QoS/QoE can be flexibly applied at the CAN layer in aggregated mode.

Figure 2 depicts an example to illustrate the principle of Internet parallelization based on VCANs, with focus on the classification process performed at ingress MANEs.

In the example given, three autonomous systems (AS) are considered, each having its own NRM and CAN Manager (CANMgr). At request of an SP entity to create VCANs, (this is the Service Manager at SP (SM@SP)) addressed to the CANMgr@AS1, three multi-domain VCANs are created spanning respectively: VCAN1: AS1, AS2; VCAN2: AS1, AS2, AS3; CAN3: AS1, AS3. The dialogue between CANMgr@AS1 and other CAN Managers to chain the VCANs in multidomain virtual networks is not represented. To simplify the example, it is supposed that the specialisation of these VCANs is performed by their Meta QoS class (MQC) of services [12-13]. 
A MQC is an abstract and flexible definition which captures a common set of QoS ranges of parameters spanning several domains. It relies on a worldwide common understanding of application QoS needs. The VCANs can be created assuming that in all AS1, 2, 3 the MQC1, 2, 3 are implemented at network level (supported by DiffServ or MPLS). Figure 2 shows the process of VCAN negotiation (action 1 on the figure) and installation in the networks (action 2). Then (action 3) MANE1 is instructed how to classify the data packets, based on information as : VCAN_Ids, Content description metadata, headers to analyse, QoS class information, policies, PHBbehaviour rules, etc. obtained from SM@SP via CANMgr@AS1 and IntraNRM@AS1 (actually this is a detail of action 2).

At its turn, the SM@SP instructs the SP/CP servers how to mark the data packets. The information to be used in content aware classification can be: high level protocol headers, content description metadata (optional), VCAN_Id (optional); statistical information extracted from the packets if no other control information is available. The data packets are analysed by the classifier, assigned and forwarded to one of the VCANs for further processing. Special algorithms are needed to reduce the amount of processing of MANE in the data plane based on deep analysis of the first packets of a flow and usage of hashing functions thereafter.

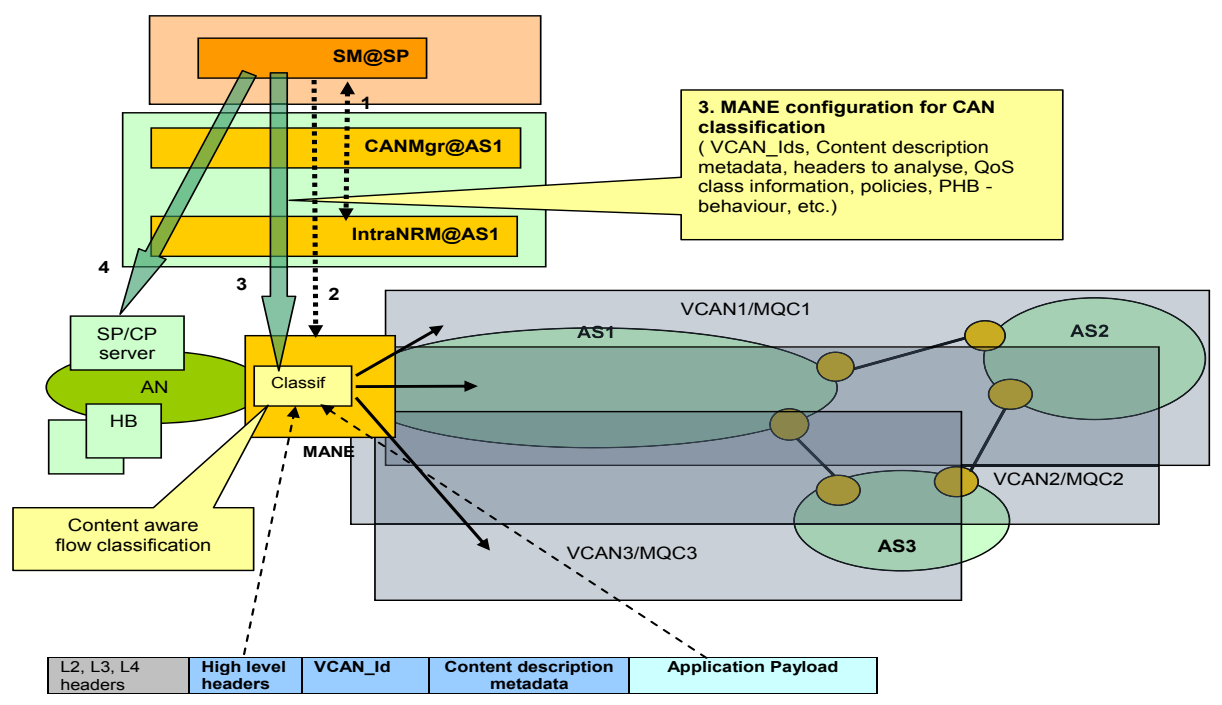

Fig. 2. Parallel VCAN creation and usage on a multi-domain infrastructure

Both traditional routing and forwarding or content/name based style can be used, in different parallel CANs due to MANE intelligence. Scalability is achieved by largely avoiding per-flow signalling in the core part of the network. In the new architecture, MANE also can act as content "caches", depending on their resource capabilities, implementing in such a way content centric capabilities. The architecture can support the client-server communication style and also P2P (between HBs) style. 
Additionally, powerful per-flow solutions (adaptation) can be applied in MANEs and $\mathrm{HBs}$, (e.g. for SVC video flows), to maintain, adapt and enhance QoE together with resource management enhancement.

The CAN management is distributed among domains. Each CAN Manager controls one or several CANs deployed in its domain. The CANMgr has interfaces with: i) HB layer and SP layer- to advertise CANs and negotiate their usage by the SP or HBs and to help at the HB layer the establishing of P2P relationships between devices of the HB layer, based on network-related distance information between these devices; ii) to the lower intra-domain network resource managers (Intra-NRM)- in order to negotiate CANs and request their installation; iii) to peer CANMgrs, in order to establish multi-domain CANs. Also CANMgr drives the monitoring at CAN layer. It is supposed that at their turn, the Intra-NRMs have established interconnection agreements at IP level. Technologies like DiffServ or MPLS can be deployed to support CAN virtual networks and offer QoS specific treatment.

\subsection{CAN Layer Security}

The aim of the security subsystem within the CAN Layer is twofold: 1) data confidentiality, integrity and authenticity; and 2) intelligent and distributed access control policy-based enforcement.

The first objective is characterized by offering, to the Service Provider (SP), a selection of three degrees of security, being: public traffic, secret content, and private communications. In public traffic no security or privacy guarantees are enforced. Secret content addresses content confidentiality and authentication by applying common cryptographic techniques over the packets' payload. Private communications is to be adopted when the confidentiality and authenticity of the entire packets, including headers, are required. The adopted strategy is to evaluate the required end-to-end security along all CAN domains and discretely apply the security mechanisms only where necessary to guarantee the required security level, with respect to the security degree invoked. The evaluation algorithm considers the user flow characteristics, CAN policies and present network conditions. In order to attain the required flexibility, the related security architecture was designed according to the hop-by-hop model [7] on top of the MANEs routers.

The second objective will pursue a content-aware approach that will be enforced by MANE routers over data in motion. Such security enforcement will be done accordingly to policies and filtering rules obtained from the CANMgr. In turn, CANMgr will compute policies and traffic filtering rules by executing security related algorithms over information gathered by the monitoring subsystem. MANE routers will derive filtering rules from packet inspection and will inform the CANMgr about those computed rules. Content-aware security technologies typically perform deep content inspection of data traversing a security element placed in a specific point in the network. The proposed approach differs by being based on MANE routers, which will be used to construct CANs.

An example of a traffic filtering rule could be to drop all traffic matching a set composed of: source IP; source port number; destination IP; destination port number. 
An example of a policy (i.e. more generic than a traffic filtering rule) would be to limit the maximum number of active connections for a given source in a period of time. Based on this policy, MANE equipments would be able to derive traffic filtering rules and to enforce them.

MANE's related security functions are then to perform attacks' identification (e.g. port-scan, IP spoofing, replay) and to enforce traffic filtering rules. CANMgr carries out collaborative work with homologous entities in order to implement access control policies definition and distribution, identify large scale attacks' (e.g. network scans based on MANE port-scan information, DDoS), and to trace back attack flow's in order to block the malicious traffic in the proximity of the source.

\section{$4 \quad$ Business Actors and Policy Implications}

A Media Ecosystem is comprised of different actors (business and/or operational entities) that interface with each other. The system architecture definition should support flexible ways of collaboration to satisfy both the end-users needs and the Service/Content/Network Providers' objectives. In ALICANTE's novel architectural paradigm, [1], the traditional content consumer can become easily a content/service provider and distributor. In this environment, the main business actors/entities envisaged (as shown in Figure 3) are the following:

Content Consumer (CC) or End-User (EU) is an entity (organisation/ individual) which may establish a contract with a Service Provider (SP) for service/content delivery.

Network Provider (NP) is the traditional entity which offers IP connectivity, providing connectivity between network domains/hosts. The NPs own and manage their IP connectivity infrastructures. The NPs may interact with each other to expand content-aware services across a larger geographical span.

Content Provider (CP) gathers/creates, maintains and releases digital content by owning and operating the network hosts (content sources) but it may not necessarily own the distributing network infrastructure.

CAN Provider (CANP) is a new ALICANTE business entity, seen as a virtual layer functionality provider. It is practically an enhanced, virtual NP. The additional CAN functions are actually performed in the network nodes, initiated by CANP but agreed by The CANP offers content-aware network services to the upper layer entities.

Service Provider (SP) is the ultimately ALICANTE business entity which is responsible for the services offered to the end-user and may interact with NPs, and/or CANPs in order to use/expand their service base.

Home-Box $(H B)$ is a new ALICANTE business entity, partially managed by the SP, the NP, and the end-user. The HBs can cooperate with SPs in order to distribute multimedia services (e.g., IPTV) in different modes (e.g. P2P).

Content is offered to the CCs or SPs through quality guarantee schemes such as Service Level Agreements (SLAs). There may be formal business agreements between CPs and NPs for hosting or co-locating CP's content servers in NPs' premises, nevertheless, an individual CC may also be a private CP. SPs aggregate content from 
multiple CPs and deliver services to CCs with higher quality. SPs may not own a transport infrastructure, but rely on the connectivity services offered by Network Providers (NPs), or CAN Providers (CANP). The SPs are ultimately responsible for the service offered to the $\mathrm{CC}$ and may interact with each other in order to expand their service base and use the services of NPs, or CANPs, via appropriate SLAs. In ALICANTE a single merged entity SP/CP is considered playing the both roles.

Each of the previously described environments is present in today actual deployments, but there is a profound limitation of collaboration among them. Typically, network providers operate separately from content providers. While content providers create and sell/release content, network providers are in charge of distributing content and ensuring a satisfactory level of QoS for the end users (by appropriating resources to network upgrades etc). User context is not taken into consideration by the Service or Content Provider (SP/CP) delivering the service (content), and therefore they are not able to deliver and adapt the service (content) to the capabilities of the end user equipment. Also, current architectures do not include any information exchange between the network and service layers, thus limiting content and context awareness in service delivery and consequently inhibiting innovation in services and applications. The real challenge and ultimate objective is to find the appropriate means for efficient cooperation between entities of the various environments to provide the end-user with the best service experience while preserving the fundamental principle of network neutrality.

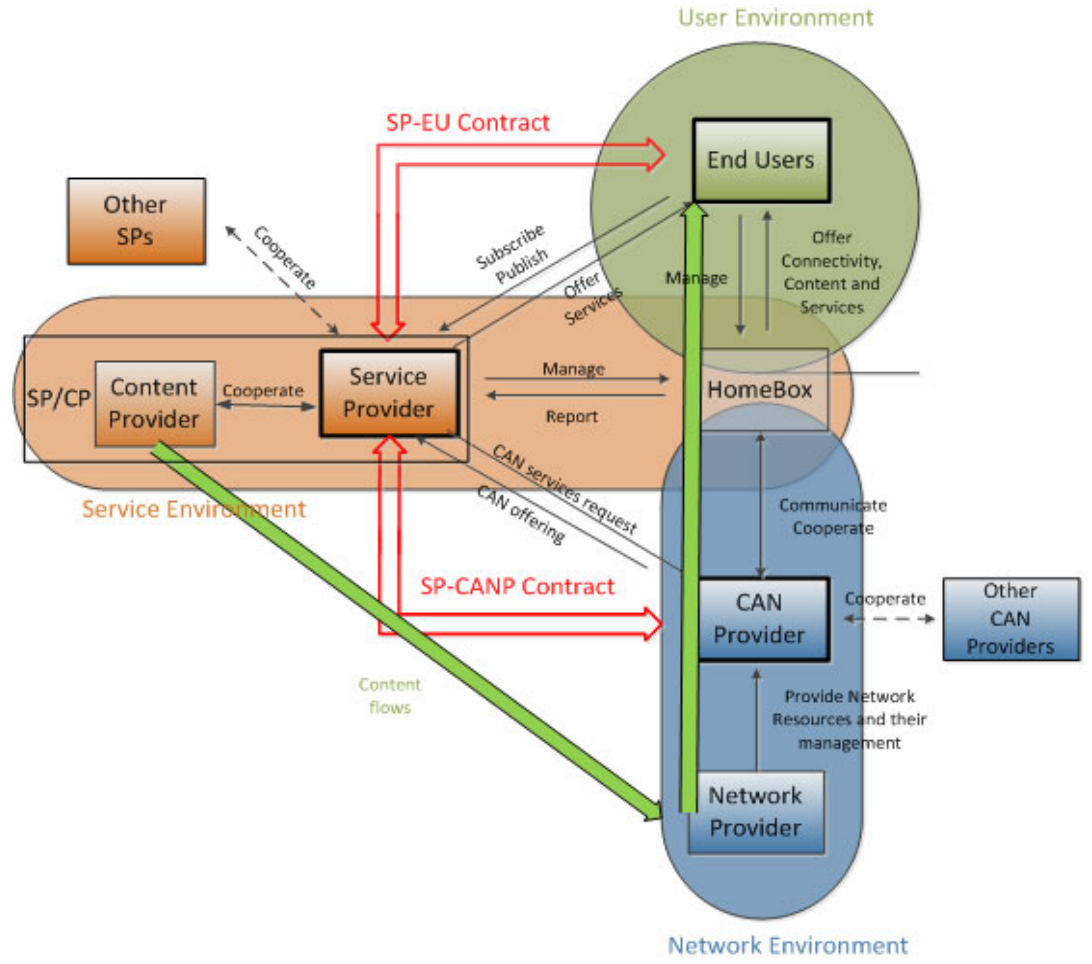

Fig. 3. ALICANTE entities and business relations 
The content-aware service delivery platform, discussed previously creates a dynamic market for network and service providers, offering the necessary network and business structures to facilitate collaboration between providers at all levels of the network/content market. This framework motivates business and service innovation in a variety of ways. It allows for revenue (and customer) sharing models between network and content providers, while preserving the upstream revenues from end-users. Also, users may influence service delivery options by requesting specific content on specific priority conditions for a specific period of time (context-awareness).

Moreover, this concept supports the creation of a 2-sided market, with network operators acting as an intermediate between content producers and content consumers, leveraging revenues from both sides. For content providers this opens a new market, characterized by specific business attributes (e.g. QoS guarantees, more intimate relation with their customers, etc.). On the user side, it increases choice and reduces switching costs between content providers (network and content layers are not integrated). In addition, the end user, as a key business actor, can effectively increase the level of choice in content and services by selecting, deploying, controlling and managing easy-to-use, affordable services and applications on service-enabled networks. Eventually the end user will have a choice of service access methods: anywhere, anytime and in any context with the appropriate awareness degree [1].

It also allows competitive content producers to enter and address niche markets. An effective scaling up of the infrastructure across multiple administrative domains (i.e. multiple NPs) could help distinguish competition in the service and network layers. Content and, most importantly, context awareness presents a possible option to address the network neutrality issue which emerges as the key issue in contemporary regulatory agendas in Europe and US. The appropriate implementation would allow management of special services and best-effort services separately. Last and not least, user privacy is a major concern since it directly relates to the consolidation of information sources where user preferences and habits may be retrieved and exploited by third parties.

\section{Conclusions}

This chapter has presented a novel Media-Ecosystem architecture, which introduces two novel virtual layers on top of the traditional Network layer, i.e. a Content-Aware Network layer (CAN) for network packet processing and a Home-Box layer for the content delivery. The innovative components, proposed to instantiate the CAN are Media-Aware Network Elements (MANE), i.e. CAN-enabled "routers" and associated managers offering together content- and context aware Quality of Service/Experience, security, and monitoring features, in cooperation with the other elements of the ecosystem. The chapter has also indicated the novel business opportunities that are created by the proposed Media-Ecosystem.

Acknowledgement. This work was supported in part by the EC in the context of the ALICANTE project (FP7-ICT-248652). 
Open Access. This article is distributed under the terms of the Creative Commons Attribution Noncommercial License which permits any noncommercial use, distribution, and reproduction in any medium, provided the original author(s) and source are credited.

\section{References}

1. FP7 ICT project: MediA Ecosystem Deployment Through Ubiquitous Content-Aware Network Environments. ALICANTE, No248652 (last accessed: March 2011) (2011), http: //www.ict-alicante.eu/

2. Borcoci, E., Negru, D., Timmerer, C.: A Novel Architecture for Multimedia Distribution based on Content-Aware Networking. In: CTRQ 2010 Conference Proceedings (2010), http: //www. iaria.org

3. Baladrón, C.: User-Centric Future Internet and Telecommunication Services. In: Tselentis, G., et al. (eds.) Towards the Future Internet, pp. 217-226. IOS Press, Amsterdam (2009)

4. Schönwälder, J., et al.: Future Internet $=$ Content + Services + Management. IEEE Communications Magazine 47(7), 27-33 (2009)

5. Zahariadis, T., et al.: Content Adaptation Issues in the Future Internet. In: Tselentis, G., et al. (eds.) Towards the Future Internet, pp. 283-292. IOS Press, Amsterdam (2009)

6. Huszák, Á., Imre, S.: Content-aware Interface Selection Method for Multi-Path Video Streaming in Best-effort Networks. In: Proc. of 16th International Conference on Telecommunications, Marrakech, Morocco, Jul. 2009, pp. 196-201 (2009)

7. Liberal, F., et al.: QoE and *-awareness in the Future Internet. In: Tselentis, G., et al. (eds.) Towards the Future Internet, pp. 293-302. IOS Press, Amsterdam (2009)

8. Martini, M.G., et al.: Content Adaptive Network Aware Joint Optimization of Wireless Video Transmission. IEEE Communications Magazine 45(1), 84-90 (2007)

9. Baker, N.: Context-Aware Systems and Implications for Future Internet. In: Tselentis, G., et al. (eds.) Towards the Future Internet, pp. 335-344. IOS Press, Amsterdam (2009)

10. Anderson, T., et al.: Overcoming the Internet Impasse through Virtualization. Computer 38(4), 34-41 (2005)

11. Chowdhury, N.M., Boutaba, R.: Network Virtualization: State of the Art and Research Challenges. IEEE Communications Magazine 47(7), 20-26 (2009)

12. Levis, P., et al.: The Meta-QoS-Class Concept: a Step Towards Global QoS Interdomain Services. Proc. IEEE, SoftCOM, Oct. 2004 (2004)

13. Paris Flegkas, et.al., Provisioning for Interdomain Quality of Service: the MESCAL Approach. IEEE Communications Magazine (June 2005)

14. Timmerer, C., et al.: Scalable Video Coding in Content-Aware Networks: Research Challenges and Open Issues. In: Proc. International Tyrrhenian Workshop on Digital Communications (ITWDC), Ponza, Italy (September 2010)

15. ITU-T SG12: Definition of Quality of Experience. TD 109rev2 (PLEN/12), Geneva, Switzerland, 16-25 Jan 2007 (2007) 